\title{
La détermination de la teneur en matière grasse des laits homogénéisés par la méthode Gerber
}

\author{
par \\ Jean PIEN
}

La méthode officielle internationale mise au point par la Fédération Internationale de Laiterie pour la détermination de la teneur en matière grasse du lait (norme $n^{\circ} 1 \mathrm{~A}-1969$ ) est basée sur la méthode de Röse-Gottlieb. Elle est parfaitement applicable à l'analyse des laits homogénéisés (pasteurisés ou stérilisés traditionnels ou stérilisés UHT).

Mais cette méthode est d'application assez longue et ne permet pas d'obtenir un résultat rapide comme cela est nécessaire dans le cas du travail industriel pour contrôler, par exemple, la marche d'un atelier de préparation de lait homogénéisé.

D'autre part, les méthodes d'extraction par solvants (comme la méthode Röse-Gottlieb) ne se prêtent pas, pour la même raison, à des contrôles en grande série, comme cela est nécessaire dans les laboratoires officiels de contrôle et même dans les laboratoires de certaines sociétés laitières importantes.

Le développement sans cesse grandissant de la production des laits stérilisés (traditionnels ou UHT) qui sont nécessairement homogénéisés et la tendance de plus en plus précise que l'on constate dans de nombreux pays d'homogénéiser également les laits pasteurisés, font que la détermination de la teneur en matière grasse par une méthode simple et rapide s'avère de plus en plus nécessaire.

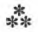

Cette méthode existe. Elle est universellement connue et appliquée : nous voulons parler de la méthode Gerber dans laquelle la 
matière grasse est libérée par un traitement acide approprié puis séparée et rassemblée dans une colonne graduée par centrifugation.

Les conditions d'application de cette méthode empirique sont purement conventionnelles. La graduation de la colonne est faite par comparaison avec les résultats de la méthode Röse-Gottlieb, de sorte que les résultats obtenus sont exacts par définition.

Mais il est évident que la méthode Gerber n'est vraiment applicable avec certitude que dans les cas pour lesquels elle a été créée et mise au point, c'est-à-dire pour l'analyse du lait entier normal non additionné de formol, non homogénéisé, etc.

$\mathrm{Si}$, donc, nous avons la certitude, maintes fois vérifiée, que la méthode Gerber donne des résultats corrects dans l'analyse du lait entier normal, on peut se demander ce qu'il en est dans le cas de l'analyse de laits non " normaux " où il serait souhaitable de pouvoir appliquer la même méthode.

Cette question se pose notamment à l'occasion de l'analyse des laits homogénéisés par la méthode Gerber. Le but du présent travail est précisément d'essayer de répondre à cette question.

L'ensemble des travaux effectués sur l'analyse des laits homogénéisés par la méthode Gerber (et notamment les nôtres) (1) ont conduit aux conclusions suivantes :

1) Dans l'analyse d'un lait homogénéisé, le résultat obtenu par la méthode Gerber est en général déficitaire et le déficit constaté varie suivant la manière dont les laits ont été homogénéisés (pression notamment).

2) Pour un lait homogénéisé, à 34 g de M.G. par litre par exemple, les chiffres trouvés par la méthode Gerber (une seule centrifugation) varient de 16 à $33 \mathrm{~g}$.

3) Il est donc nécessaire de procéder à plusieurs centrifugations successives. Après 3 ou 4 centrifugations, le résultat obtenu paraît constant et est égal ou légèrement supérieur au chiffre réel.

Une première constatation, très importante, est donc que, dans de nombreux laits homogénéisés (notamment ceux qui ont subi une pression d'homogénéisation très élevée et qui, de ce fait contiennent une grande quantité de globules gras extrêmement fins), la première cen-

(1) Annales des Falsifications et de l'Expertise chimique, 1966, p. 145-150. 
trifugation conduit à un résultat inférieur au chiffre réel. Ce phénomène s'explique aisément de la manière suivante :

La matière grasse du lait (homogénisé ou non) est constituée par une émulsion formée de globules gras entourés d'une double membrane protectrice. L'acide sulfurique, mélangé au lait dans le butyromètre, détruit ces membranes et libère les globules gras. Or, avant que cette matière grasse libérée ne se rassemble en une masse continue, les micro-gouttelettes dont elle est constituée possèdent encore une certaine individualité qui s'oppose à leur drainage par centrifugation et cela d'autant plus qu'elles sont plus fines. Suivant la loi de Stokes, en effet, la vitesse de leur cheminement centripète est proportionnelle au carré de leur diamètre ; de sorte que la vitesse de translation d'un globule gras de $1 \mu$, qui représente la majorité des globules gras du lait homogénéisé, est 100 fois plus faible que celle d'un globule gras de $10 \mu$ (diamètre moyen des globules gras du lait normal).

Il est donc clair que les globules gras les plus fins, ne sont rassemblés qu'avec un certain retard et l'on comprend qu'au cours d'une première centrifugation, les résultats obtenus puissent être inférieurs à la réalité.

Une deuxième constatation, corollaire de la précédente, et d'ailleurs bien connue de tous les laboratoires de laiterie, réside dans le fait qu'à la suite de plusieurs centrifugations successives, le résultat augmente et semble se stabiliser sur un palier obtenu après un nombre de centrifugations, variable suivant les laits, généralement compris entre 2 et 4 .

Il convient de signaler que ce « palier » est pratiquement toujours un peu supérieur au chiffre réel (de 0,5 à $1 \mathrm{~g}$ de M.G. par litre, quelquefois plus). Voilà pourquoi nous avons pu dire que, "dans le cas du dosage, par la méthode Gerber, de la matière grasse des laits homogénéisés, il était nécessaire de procéder à plusieurs centrifugations successives jusqu'à l'obtention d'un chiffre constant, qui pouvait être tenu pour exact ou voisin de la réalité ».

Tous les traités d'analyse et même certains textes officiels tiennent le même langage. On pourrait donc considérer le problème comme pratiquement résolu.

Or il se trouve que nous avons maintenant de nouvelles raisons de penser que ce problème se pose encore et qu'il risque, dans certains cas, de conduire à de graves erreurs. En effet :

Ayant constaté à maintes reprises que les résultats trouvés après plusieurs centrifugations étaient un peu supérieurs au chiffre réel, 
nous avons eu l'idée de poursuivre ces centrifugations jusqu'à la $20^{\text {e }}$ et voici ce que nous avons constaté après homogénéisation de laits dont la teneur initiale en matière grasse était égale à $34 \mathrm{~g}$ par litre :

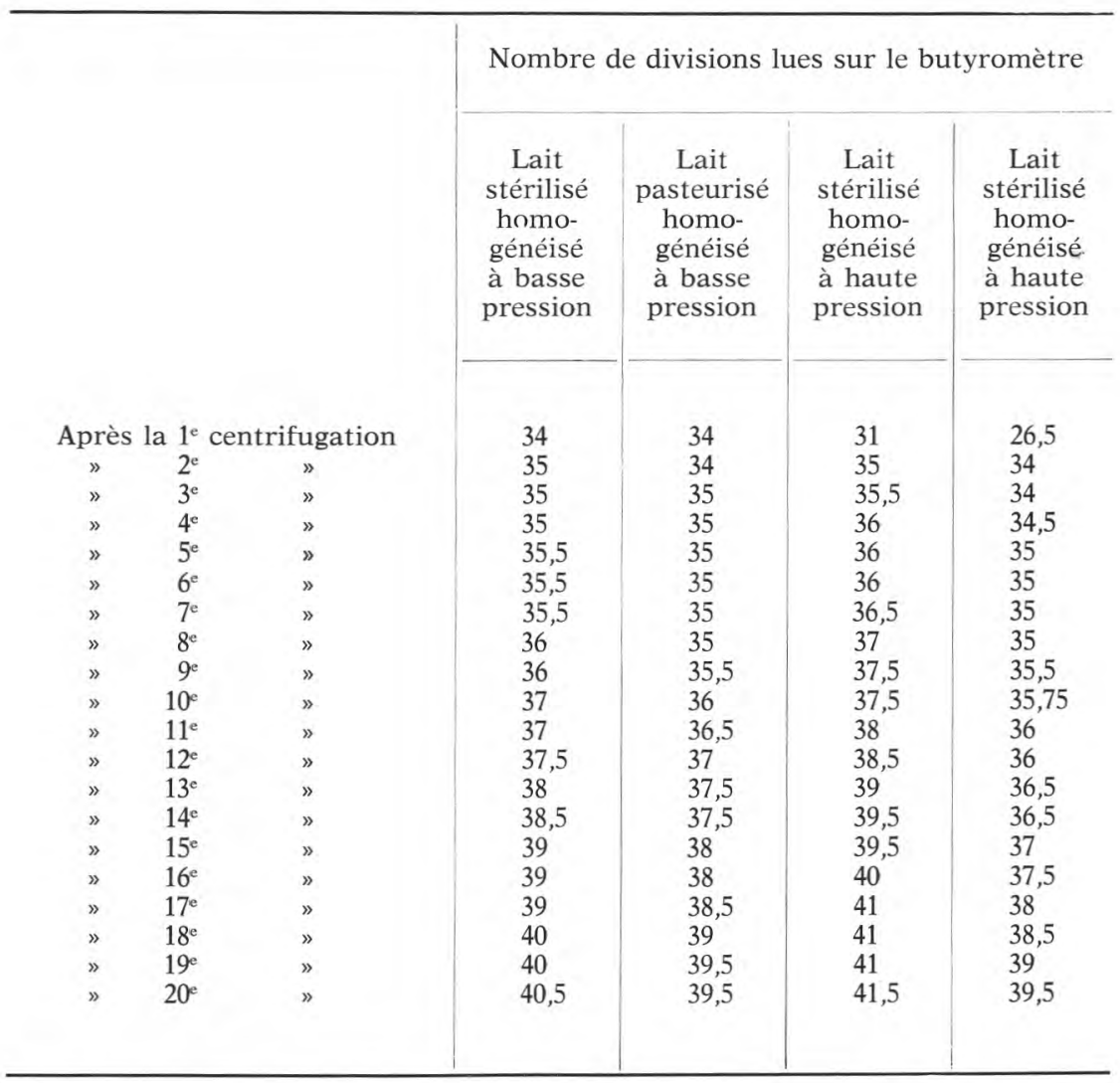

\section{Conclusions}

1) Nous retrouvons, au cours des premières centrifugations, l'existence d'un plateau correspondant ici aux chiffres de 34 ou $35 \mathrm{~g}$ par litre, qui commence à la $2^{\mathrm{e}}$ ou à la $3^{\mathrm{e}}$ centrifugation et semble stabilisé pendant 3 ou 6 centrifugations - ce qui peut faire croire que le résultat définitif (un peu supérieur au chiffre réel) est atteint.

2) Mais, en poursuivant les centrifugations, nous constatons que le résultat augmente sans cesse jusqu'à atteindre le chiffre de $40 \mathrm{~g}$ par litre. Il est bien évident que cet excès de $6 \mathrm{~g}$ de M.G. par 
litre ne correspond plus à un complément d'extraction de globules gras trop fins, mais à une modification de la composition de la matière grasse qui lui confère un volume de plus en plus grand.

3) Donc cette anomalie grave ne provient pas d'un excès de centrifugation, mais simplement d'un excès de durée de contact de la M.G. avec l'acide sulfurique et l'alcool amylique, dans les conditions thermiques qui sont celles de l'essai.

Pour confirmer cette conclusion vraisemblable, nous avons, sur les mêmes laits, procédé à des centrifugations séparées par $1 \mathrm{~h}$ de séjour au bain-marie à $67^{\circ} \mathrm{C}$.

Nous avons abouti aux mêmes résultats : après $5 \mathrm{~h}$ de séjour au bain-marie à $67^{\circ} \mathrm{C}$ (c'est-à-dire après 6 centrifugations seulement) nous avons obtenu sur ces mêmes laits des résultats compris entre 40 et $41 \mathrm{~g}$ de matière grasse par litre.

Il se confirme donc que ce n'est pas le nombre de centrifugations qui est à l'origine de l'accroissement constaté des résultats, mais la durée de contact matière grasse / acide sulfurique / alcool amylique à $67^{\circ} \mathrm{C}$.

4) Dans le cas des laits homogénéisés à haute pression (plus de $200 \mathrm{~kg} / \mathrm{cm}^{2}$ ) la $1^{\text {e }}$ centrifugation donne un résultat inférieur au chiffre réel. Mais dès la $2^{\mathrm{e}}$ centrifugation ce chiffre réel est atteint ou dépassé et à la $4^{\mathrm{e}}$ ou $5^{\mathrm{e}}$ centrifugation on atteint un plateau de 35 ou $36 \mathrm{~g}$ qui se maintient pendant plusieurs centrifugations.

\section{噒}

Au point où nous en sommes de cet exposé, nous avons acquis les certitudes suivantes :

1) En multipliant le nombre de centrifugations ou, ce qui revient au même, en prolongeant la durée de séjour des butyromètres au bain-marie à $67^{\circ} \mathrm{C}$, c'est-à-dire la durée de contact de la M.G. avec les réactifs dans le butyromètre, on constate une augmentation $d u$ volume de la M.G. qui fausse les résultats par excès.

2) Cette altération progressive est relativement lente lors des premières centrifugations et fait apparaître un plateau que l'on a longtemps considéré comme correspondant au résultat exact ou à un chiffre peu différent.

3) A la lumière de ce que nous savons maintenant, nous pouvons affirmer que lorsque ce premier plateau se situe au-dessus du chiffre réel, on assiste déjà à un commencement d'altération de la M.G. et, donc, à un début d'erreur par excès. (Dans le cas d'un plateau se situant exactement à $34 \mathrm{~g}$ par exemple, il est très vraisemblable que l'on se trouve en présence d'une compensation d'erreurs : extraction encore un peu incomplète de globules gras très fins, 
compensée par un début d'altération avec excès de volume. Un peu plus tard, au cours des centrifugations suivantes, seule l'altération de la M.G. continue à se manifester en provoquant une erreur par excès).

Nous verrons plus loin quelles conclusions pratiques nous devrons tirer de toutes ces constatations.

Auparavant, nous devons cependant nous demander quel est le mécanisme de ce phénomène afin de pouvoir, si possible, y porter remède et rendre la méthode Gerber enfin applicable en toute sécurité aux laits homogénéisés.

Dans la recherche des causes profondes de cet accroissement de volume de la M.G. au cours de séjours prolongés au bain-marie à $67^{\circ} \mathrm{C}$, nous pouvons envisager plusieurs hypothèses :

- Combinaison de la M.G. avec l'acide sulfurique (par l'intermédiaire d'une hydrolyse d'une partie des glycérides, formation d'un ester sulfurique du glycérol qui se dissoudrait dans l'excès de M.G. non-hydrolysée).

- Combinaison de l'alcool amylique avec l'acide sulfurique et formation de sulfate d'amyle soluble dans la M.G. (1).

- Dissolution pure et simple de l'alcool amylique dans la M.G.

Dans les trois hypothèses, on peut s'attendre à une augmentation du volume de la M.G. séparée.

Mais, surtout dans le cas des deux premières, on peut admettre que ces réactions sont fortement influencées par la température. Leur vitesse, suivant la règle générale des $\mathrm{Q}_{10}$, doit vraisemblablement être doublée ou triplée pour chaque élévation de $10^{\circ} \mathrm{C}$. Cette remarque nous mène directement à l'idée que si la température du bain-marie pouvait être descendue de $10^{\circ} \mathrm{C}$ ou même de $20^{\circ} \mathrm{C}$, la vitesse des réactions présumées ci-dessus serait divisée par 3 ou par 9 , ce qui reviendrait peut-être à les annuler pratiquement.

Nous avons donc entrepris des recherches dans cette direction, et avons tout d'abord choisi d'opérer à $40^{\circ} \mathrm{C}$ parallèlement à la température habituelle de $67^{\circ} \mathrm{C}$. Voici les résultats obtenus sur un lait homogénéisé au cours des 20 centrifugations successives auxquelles nous l'avons soumis (lait titrant 34,7 g de M.G. par litre) :

(1) Mme KuzDzal-Savore, dans les travaux qu'elle a effectués pour le CNERNA concernant la composition de la matière grasse extraite dans les diverses méthodes d'analyse, a montré que la M.G. extraite du Gerber renfermait des quantités importantes d'esters iso-amyliques (13 à 14 p. 100) à côté de diglycérides et d'acides gras libres résultant de l'hydrolyse de la matière grasse. 


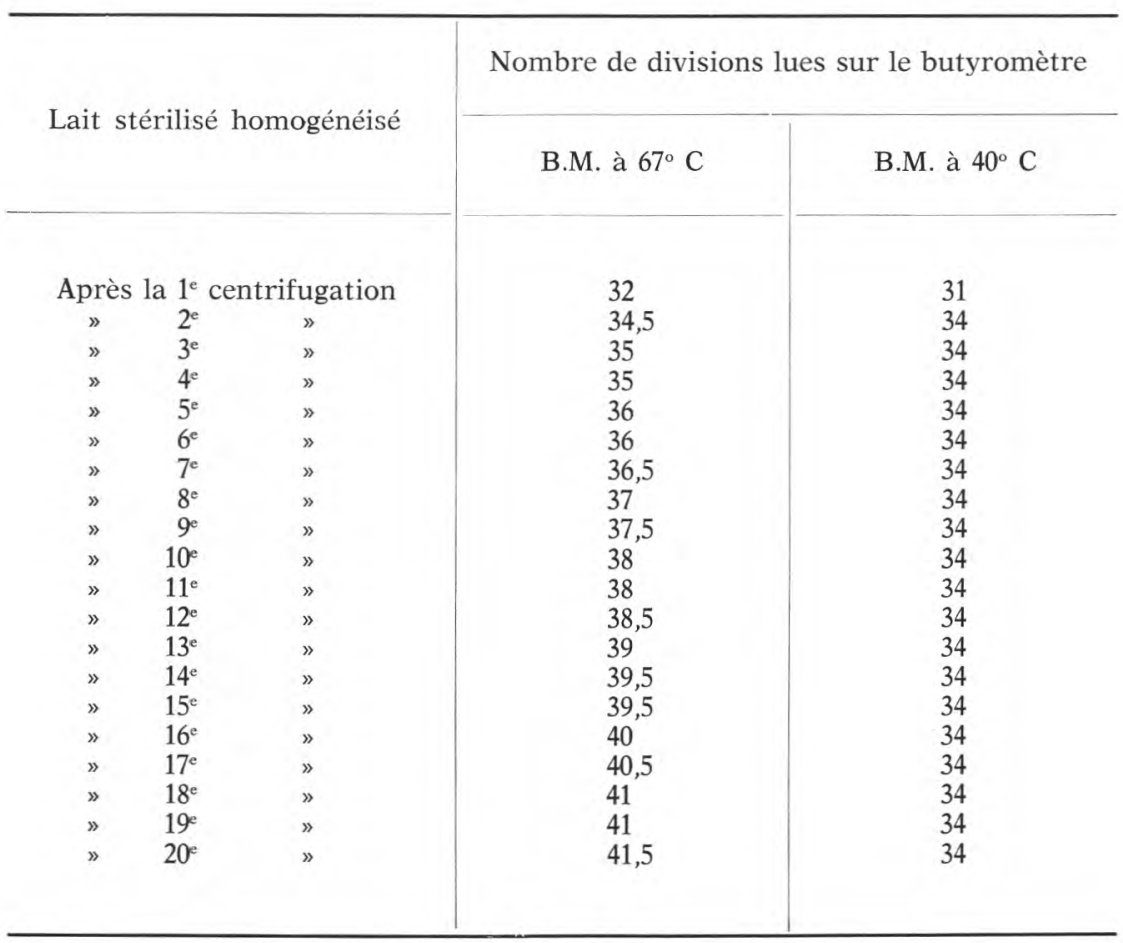

\section{Conclusions}

1) Nous retrouvons, en faisant usage du bain-marie à $67^{\circ} \mathrm{C}$, la même évolution de la M.G. séparée, dans le sens d'une augmentation de volume qui fausse les déterminations par excès.

2) En faisant séjourner les butyromètres dans un bain-marie à $40^{\circ} \mathrm{C}$ entre les centrifugations, nous constatons que le chiffre de 34 (1) est atteint dès la $2^{\mathrm{e}}$ centrifugation et que ce résultat n'est pas modifié au cours des centrifugations ultérieures.

3) Ce résultat, extrêmement important, nous fournit à la fois l'explication des phénomènes constatés et, peut-être, le remède à leur apporter.

Cette expérience a été répétée sur divers laits homogénéisés et s'est trouvée confirmée. Nous avons voulu cependant voir ce qui se passe dans le cas d'un lait non-homogénéisé (simplement pasteurisé) et voici ce que nous avons obtenu (cas d'un lait pasteurisé à $34 \mathrm{~g}$ de M.G. par litre) :

(1) Correspondant à la teneur réelle de 34,7 (voir ci-après). 


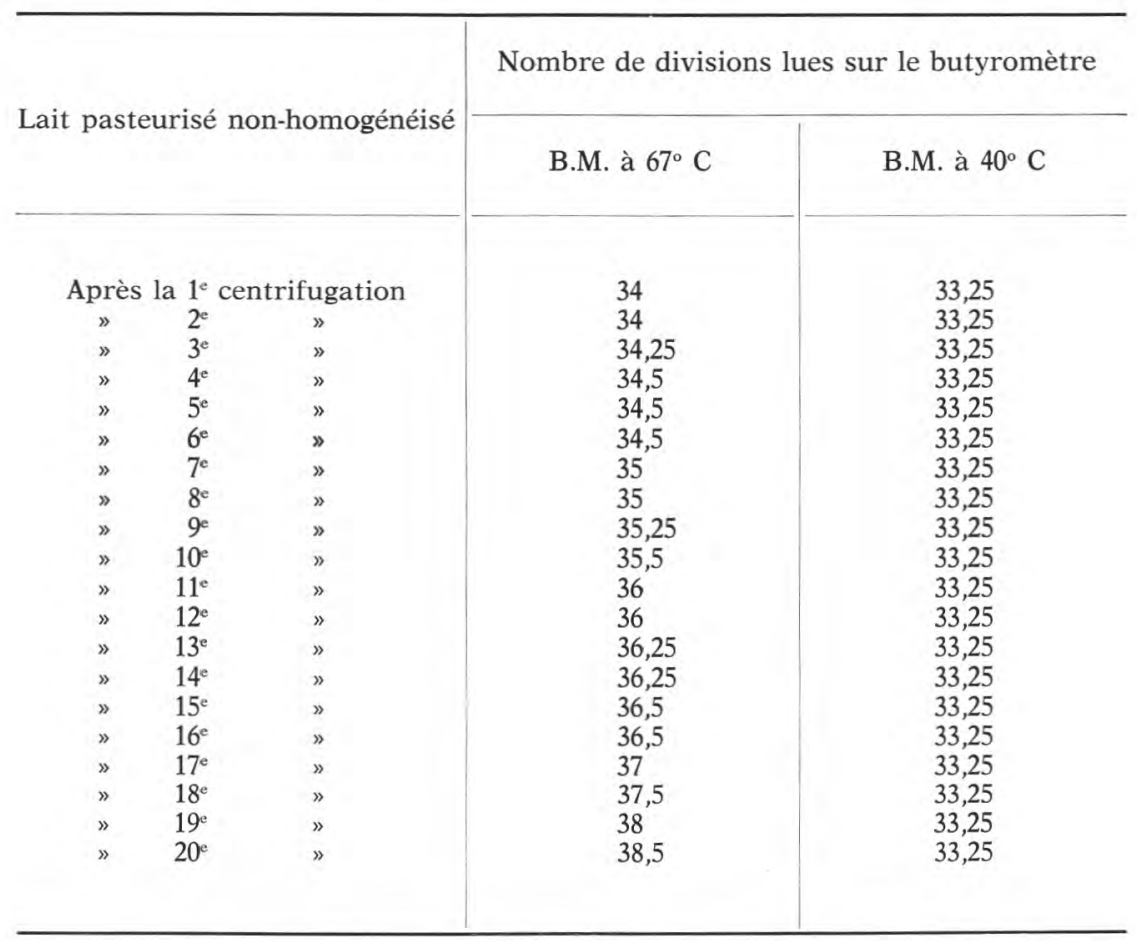

\section{Conclusions}

1) A $67^{\circ} \mathrm{C}$ le lait pasteurisé non-homogénéisé se comporte un peu différemment du lait homogénéisé. La première centrifugation donne d'emblée le chiffre réel (ce qui est normal puisque l'on applique là les conditions réelles de la méthode Gerber).

2) $\mathrm{A} 40^{\circ} \mathrm{C}$ on obtient, à la première centrifugation, un chiffre un peu inférieur au chiffre réel (qui est celui de la première centrifugation à $67^{\circ} \mathrm{C}$ ) et ce résultat n'est pas modifié au cours des centrifugations successives.

Il est normal que, pour une même masse de M.G., le volume occupé à $40^{\circ} \mathrm{C}$, c'est-à-dire le nombre de divisions, soit inférieur au volume ou au nombre de divisions occupés à $67^{\circ} \mathrm{C}$.

Nous avons effectué le calcul de ce volume à $40^{\circ} \mathrm{C}$ d'une masse de M.G. correspondant à 34 divisions lues à $67^{\circ} \mathrm{C}$. Ce travail est assez complexe car il suppose connues les masses volumiques de la M.G. séparée à diverses températures pour aboutir à la connaissance du coefficient de dilatation de cette matière grasse (qui, pour nous, vaut 0,0007963). Il nécessite ensuite l'utilisation des formules permettant de 
calculer le volume d'une certaine masse de M.G. à une température donnée, en fonction du volume qu'elle occupe à une autre température. D'autre part, il faut calculer les différences de dilatation du "récipient ", c'est-à-dire de la colonne graduée à ces deux températures pour corriger les volumes précédents.

Nous publierons ultérieurement le résultat de ces divers travaux et calculs.

Disons seulement qu'une masse de M.G. pure de $374 \mathrm{mg}$, représentant exactement 34 divisions à $67^{\circ} \mathrm{C}$ occupe un volume de $0,42353 \mathrm{~cm}^{3}$. Cette même masse occupe, à $40^{\circ} \mathrm{C}$, un volume absolu de $0,41489 \mathrm{~cm}^{3}$, inférieur au précédent à cause de la contraction, mais majoré d'une certaine quantité pour tenir compte de la différence de dilatation du verre (plus faible à $40^{\circ} \mathrm{C}$ qu'à $67^{\circ} \mathrm{C}$ ) et aboutir à un « volume apparent » de $0,41519 \mathrm{~cm}^{3}$ à $40^{\circ} \mathrm{C}$.

Tous calculs faits, cette masse de M.G. correspondant à 34 divisions à $67^{\circ} \mathrm{C}$, se traduira, à $40^{\circ} \mathrm{C}$, par la lecture de 33,33 divisions. Le facteur par lequel il faut multiplier la lecture faite à $67^{\circ} \mathrm{C}$ pour découvrir le chiffre correspondant à $40^{\circ} \mathrm{C}$, est égal à 0,9803 . Inversement, connaissant la lecture à $40^{\circ} \mathrm{C}$, il faut multiplier ce résultat par 1,020 pour découvrir la valeur du résultat qu'on aurait obtenu en travaillant à $67^{\circ} \mathrm{C}$.

Application pratique : un lait dont la teneur exacte est de $34 \mathrm{~g}$ par litre à $67^{\circ} \mathrm{C}$, doit donner, à $40^{\circ} \mathrm{C}$, le chiffre théorique de $33,33 \mathrm{~g}$. Cela signifie (dans la méthode Gerber) que l'on fera une lecture comprise entre $331 / 4$ et 33,5 . (Inversement, le résultat, lu à $40^{\circ} \mathrm{C}$, de $331 / 4$ ou de 33,5 donnera, multiplié par 1,02, les chiffres suivants: 33,92 ou 34,17 comme résultat réel supposé lu à $67^{\circ} \mathrm{C}$ ).

3) Il est donc possible, en travaillant à $40^{\circ} \mathrm{C}$, d'effectuer plusieurs centrifugations successives, pour obtenir l'extraction complète dans le cas des laits homogénéisés, sans risquer de voir le résultat devenir faux par excès comme dans le cas du travail à $67^{\circ} \mathrm{C}$. Nous avons donc là le moyen de doser avec certitude la teneur en M.G. des laits homogénéisés. Cependant en travaillant à $40^{\circ} \mathrm{C}$, il faudrait des butyromètres spéciaux gradués pour cette température. A défaut de ces appareils nouveaux, il est possible d'utiliser les butyromètres classiques et de multiplier le résultat obtenu par le coefficient de correction : 1,02.

Existe-t-il, entre $40^{\circ} \mathrm{C}$ et $67^{\circ} \mathrm{C}$, une autre température qui ne fausserait pas le volume de la M.G. séparée? 
Nous avons eu la chance, en travaillant à $40^{\circ} \mathrm{C}$, d'obtenir d'emblée l'excellent résultat que nous espérions. Nous devions alors nous demander s'il était possible de relever cette température pour essayer de réduire (voire de supprimer) le facteur de correction.

Les expériences que nous avons effectuées dans cette direction nous ont apporté la preuve qu'une température intermédiaire entre $67^{\circ} \mathrm{C}$ et $40^{\circ} \mathrm{C}$ (notamment celle de $50^{\circ} \mathrm{C}$ ) n'est pas utilisable car elle entraîne des altérations progressives de la M.G., faussant le résultat par excès, comme dans le cas de la température de $67^{\circ} \mathrm{C}$ (mais à un moindre degré). C'est donc la température de $40^{\circ} \mathrm{C}$ qu'il convient d'employer pour éviter une altération de la M.G. au cours des centrifugations successives.

L'emploi de cette température nécessite l'application du coefficient 1,02 au résultat obtenu pour avoir le chiffre réel.

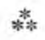

Dans le but de confirmer pleinement les conclusions auxquelles nous avons abouti au cours de ce travail, nous avons encore effectué l'expérience suivante : nous prenons du lait pasteurisé, non-homogénéisé, et nous dosons sa M.G. selon Gerber aux deux températures de $40^{\circ} \mathrm{C}$ et $67^{\circ} \mathrm{C}$, en poursuivant les séjours au bain-marie et les centrifugations pendant plusieurs heures; puis nous homogénéisons ce lait à haute pression et nous recommençons les dosages de M.G. dans les mêmes conditions que dans le cas du lait examiné avant homogénéisation.

Voici les résultats obtenus :

\begin{tabular}{|c|c|c|c|c|}
\hline & \multicolumn{4}{|c|}{ Nombre de divisions lues sur le butyromètre } \\
\hline & \multicolumn{2}{|c|}{$\begin{array}{l}\text { Lait pasteurisé } \\
\text { avant homogénéisation }\end{array}$} & \multicolumn{2}{|c|}{$\begin{array}{l}\text { Même lait } \\
\text { après homogénéisation }\end{array}$} \\
\hline & à $67^{\circ} \mathrm{C}$ & à $40^{\circ} \mathrm{C}$ & à $67^{\circ} \mathrm{C}$ & à $40^{\circ} \mathrm{C}$ \\
\hline 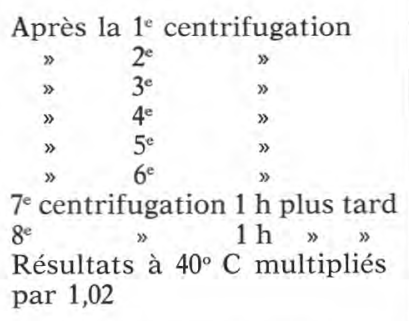 & $\begin{array}{l}34 \\
34 \\
34 \\
34,5 \\
35 \\
35 \\
36 \\
36,5 \\
-\end{array}$ & $\begin{array}{l}33,5 \\
33,5 \\
33,5 \\
33,5 \\
33,5 \\
33,5 \\
33,5 \\
33,5 \\
34,17\end{array}$ & $\begin{array}{l}28 \\
34 \\
34,5 \\
35 \\
35 \\
35 \\
36 \\
37 \\
-\end{array}$ & $\begin{array}{l}25 \\
33,5 \\
33,5 \\
33,5 \\
33,5 \\
33,5 \\
33,5 \\
33,5 \\
34,17\end{array}$ \\
\hline
\end{tabular}




\section{Conclusion}

Il est clairement confirmé que l'emploi de la température de $67^{\circ} \mathrm{C}$ ne permet pas de faire plusieurs centrifugations successives sans risquer de voir les résultats faussés par excès. En revanche, en utilisant la température de $40^{\circ} \mathrm{C}$ pour l'analyse des laits homogénéisés, on peut effectuer de nombreuses centrifugations sans risquer de fausser le résultat par excès. Dès qu'un chiffre parfaitement stable est obtenu, il peut être considéré comme correct. Il suffit alors de le multiplier par 1,02 pour obtenir le résultat réel correspondant à la température de $67^{\circ} \mathrm{C}$.

\section{腣}

\section{Conclusions générales}

1) Lorsqu'on dose la M.G. des laits homogénéisés par la méthode Gerber classique, il est nécessaire de procéder à plusieurs centrifugations successives pour rassembler la totalité de la M.G. dans la colonne graduée du butyromètre.

2) Mais, ce faisant, on provoque un accroissement du volume de la M.G. séparée, ce qui conduit à des résultats faussés par excès.

3) Cette augmentation de volume se poursuit avec le nombre de centrifugations ou avec la durée de séjour des butyromètres à $67^{\circ} \mathrm{C}$.

4) Elle est due, non pas à la centrifugation, mais au séjour des butyromètres dans le bain-marie à $67^{\circ} \mathrm{C}$ par suite d'une altération de la M.G. ou de la formation d'esters amyliques qui se dissolvent dans la M.G. et en augmentent le volume.

5) Si les séjours au bain-marie ont lieu dans une eau à $40^{\circ} \mathrm{C}$, le volume de la M.G. n'est pas modifié, quels que soient les temps de séjour et le nombre de centrifugations. Cette circonstance permet donc de rassembler la totalité de la M.G. des laits homogénéisés sans risque d'augmentation du volume de la M.G. séparée.

6) Le volume de la M.G. séparée à $40^{\circ} \mathrm{C}$ est évidemment inférieur, pour une même masse de M.G., à celui qui est normalement séparé à $67^{\circ} \mathrm{C}$. Il faut donc corriger le chiffre lu à $40^{\circ} \mathrm{C}$ en le multipliant par le facteur 1,02, afin d'obtenir le résultat réel qui serait celui que l'on obtiendrait à $67^{\circ} \mathrm{C}$ avec le même lait non-homogénéisé.

D'où la méthode de dosage de la M.G. des laits homogénéisés :

1) Opérer exactement comme dans le cas de l'analyse des laits ordinaires à cette seule différence près que le bain-marie, au lieu d'être réglé à $67^{\circ} \mathrm{C}$, sera porté et maintenu à $40^{\circ} \mathrm{C}$.

2) Procéder à plusieurs centrifugations successives (entrecoupées de séjours de $5 \mathrm{mn}$ au bain-marie) jusqu'à obtention d'un résultat constant.

3) Multiplier le résultat obtenu par le facteur 1,02. 
Le chiffre final ainsi corrigé est pratiquement identique à celui que l'on aurait obtenu, sur le même lait avant homogénéisation, en appliquant la méthode Gerber classique, c'est-à-dire avec une seule centrifugation suivie d'un séjour de $5 \mathrm{mn}$ au bain-marie à $67^{\circ} \mathrm{C}$.

Reçu pour publication le 9 janvier 1974.

\section{R és u m é}

Lorsqu'on dose la matière grasse des laits homogénéisés par la méthode Gerber classique, il est nécessaire de procéder à plusieurs centrifugations successives, entrecoupées de séjours de $5 \mathrm{mn}$ au bainmarie à $65^{\circ} \mathrm{C}-67^{\circ} \mathrm{C}$, pour extraire la totalité de la matière grasse de la prise d'essai et la rassembler dans la colonne graduée du butyromètre.

Mais, ce faisant, on provoque un accroissement progressif du volume de la matière grasse, ce qui conduit à des résultats faussés par excès. Il est d'ailleurs impossible d'obtenir un résultat vraiment constant, le chiffre lu augmentant sans cesse avec le nombre de séjours au bain-marie. Cet accroissement progressif du volume de la matière grasse est dû à la formation d'esters amyliques qui se dissolvent dans la matière grasse.

Cette cause d'erreur est supprimée si les séjours au bain-marie ont lieu dans de l'eau à $40^{\circ} \mathrm{C}$ au lieu de $65^{\circ} \mathrm{C}-67^{\circ} \mathrm{C}$. On parvient alors après quelques centrifugations, à un résultat réellement constant. Il suffit de multiplier le chiffre obtenu par le facteur 1,02 pour avoir la teneur réelle en matière grasse du lait homogénéisé.

\section{S u m m a r y}

When we have to determine the fat content of homogenized milks, by the classical Gerber method, it is necessary to proceed to several consecutive centrifugations, separated by immersions of $5 \mathrm{mn}$ in a water bath at $65^{\circ} \mathrm{C}-67^{\circ} \mathrm{C}$, to extract the whole fat and collect it in the graduated column of the butyrometer.

But, this procedure results in a progressive increase of the volume of the fat, which leads to false results by excess. Moreover, it is impossible to reach a really constant result, because the figures read on the butyrometer grow inceasingly with the number of immersions in the water-bath. This progressive increase of the fat volume is caused by the production of amyl-esters which dissolve in the fat.

This cause of error is suppressed if the immersions in the waterbath are made in water at $40^{\circ} \mathrm{C}$ instead of $65^{\circ} \mathrm{C}-67^{\circ} \mathrm{C}$. Thus, after a few centrifugations, a really constant result is obtained. It suffices, then, to multiply this figure by the factor 1,02 to have the true fat content of the homogenized milk. 